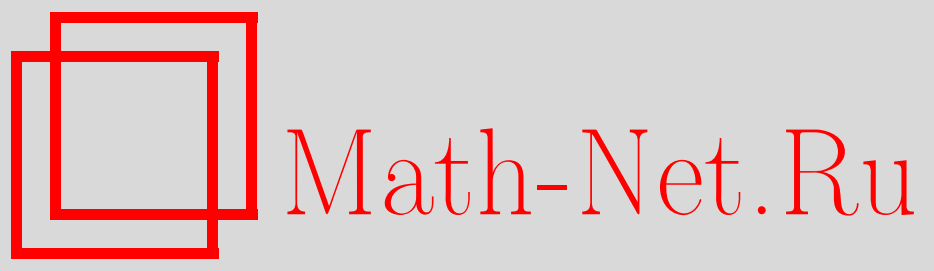

П. Г. Гриневич, П. М. Сантини, Фазовые резонансы для повторяемости аномальных волн НУШ в квазисимметричном случае, $Т М \Phi, 2018$, том 196, номер 3, 404-418

DOI: https://doi.org/10.4213/tmf9544

Использование Общероссийского математического портала Math-Net.Ru подразумевает, что вы прочитали и согласны с пользовательским соглашением http://www.mathnet.ru/rus/agreement

Параметры загрузки:

IP: 54.210 .77 .194

26 апреля 2023 г., 10:54:49

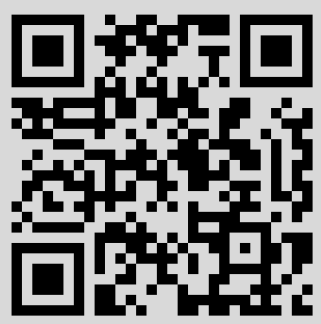




\title{
ФАЗОВЫЕ РЕЗОНАНСЫ \\ ДЛЯ ПОВТОРЯЕМОСТИ АНОМАЛЬНЫХ ВОЛН НУШ В КВАЗИСИММЕТРИЧНОМ СЛУЧАЕ
}

\begin{abstract}
Наши недавние аналитические результаты о задаче Коши для фокусируюшего нелинейного уравнения Шредингера с начальными данными в виде периодического возмущения неустойчивого фона позволяют в простейшем случае одной неустойчивой моды получить полное аналитическое описание повторяемости аномальных волн. С учетом того, что современные эксперименты в волновых бассейнах и нелинейной оптике, посвященные наблюдению повторяемости Ферми-Паста-Улама, осуществляются в почти симметричных конфигурациях, с использованием полученных ранее результатов выведены полезные аналитические формулы для фазовых резонансов в повторяемости для почти симметрического случая, которые можно сопоставить с данными экспериментов.
\end{abstract}

Ключевые слова: фокусирующее нелинейное уравнение Шредингера, аномальные волны, повторяемость, почти симметричные конфигурации, фазовые резонансы.

DOI: https://doi.org/10.4213/tmf9544

\section{1. ВВЕДЕНИЕ}

Фокусирующее нелинейное уравнение Шредингера (НУШ)

$$
i u_{t}+u_{x x}+2|u|^{2} u=0, \quad u=u(x, t) \in \mathbb{C},
$$

является универсальной моделью, описывающей распространение квазимонохроматических волн в слабо нелинейных средах, включая глубокую воду [1], нелинейную оптику [2]-[4], ленгмюровские волны в плазме [5] и конденсат Бозе-Эйнштейна

Работа П. Г. Гриневича поддержана ФАНО России (тема № 0033-2018-0009), а также Sezione di Roma INFN и проектом Sapienza Ateneo 2017 г.

${ }^{*}$ Институт теоретической физики им. Л. Д. Ландау Российской академии наук, Черноголовка, Московская обл., Россия. E-mail: pgg@landau.ac.ru

${ }^{\dagger}$ Московский государственный университет им. М. В. Ломоносова, Москва, Россия

‡Dipartimento di Fisica, Università di Roma "La Sapienza", Roma, Italy

$\S$ Istituto Nazionale di Fisica Nucleare, Sezione di Roma, Roma, Italy.

E-mail: paolo.santini@roma1.infn.it 
с притяжением [6]. Хорошо известно, что его элементарное решение

$$
u_{0}(x, t)=e^{2 i t},
$$

описывающее волны Стокса [7] на воде, состояние с постоянной интенсивностью света в нелинейной оптике и состояние с постоянной плотностью бозонов в конденсате Бозе-Эйнштейна, неустойчиво относительно возмущений с достаточно большой длиной волны [1], [8]-[12], и данная модуляционная неустойчивость (MH) считается основным механизмом образования аномальных волн (AВ, экстремальных волн, волн-убийц) в природе [13]-[18]. АВ - это короткоживущие волны, возникающие как будто бы из ниоткуда в данных физических системах.

Интегрируемость НУШ [19] позволяет строить решения, отвечающие малым возмущениям постоянного фона, с помощью вырождения конечнозонных решений (см. работы [20]-[23]), при котором спектральная кривая вырождается в рациональную, либо напрямую с использованием классического преобразования Дарбу [24], [25] или метода одевания [26], [27]. В числе этих базисных решений мы хотели бы упомянуть решения Перегрина [28], рационально убывающие по $x$ и $t$ относительно фона (2), так называемые солитоны Кузнецова-Каваты-Иноуе-Ма [29]-[31], экспоненциально локализованные в пространстве относительно фона и периодические по времени, а также решения, найденные Ахмедиевым, Элеонским и Кулагиным в статье [32], периодические по $x$ и экспоненциально локализованные по времени относительно фона (2), известные в литературе как бризеры Ахмедиева, равно как и их эллиптические аналоги [33], [34]. Односолитонные решения более общего вида на фоне (2) (см., например, работы [20], [35]) отвечают спектральному параметру в точках общего положения. Эти решения обобщаются на многосолитонные, описывающие нелинейное взаимодействие солитонов (см., например, работы [20], [36]-[39]). Отметим, что решения Перегрина являются строго гомоклинными и описывают АВ, появляющиеся почти из ниоткуда и в будущем исчезающие, тогда как многосолитонные решения ахмедиевского типа являются почти гомоклинными, поскольку они возвращаются к фону, отличающемуся от исходного на фазовый множитель. Также были построены обобщения этих решений для интегрируемого многокомпонентного НУШ (см., например, работы [40]-[42]).

Естественным образом возникает вопрос, насколько хорошо вышеупомянутые солитонные решения фокусирующего НУШ на фоне (2) соответствуют решениям задачи Коши в осмысленной постановке, и если ответ нас устраивает, то каков механизм их появления в общих ситуациях.

Что касается задачи Коши для фокусирующего НУШ в предположении, что начальное условие является локализованным возмущением фона, то медленно модулированные периодические колебания, описываемые эллиптическими решениями (1), играют существенную роль при описании поведения на больших временах [43], [44]. Для того чтобы исследовать этот базисный вопрос в пространственно-периодической постановке, мы недавно построили решение $x$-перидической задачи Коши для НУШ с точностью до поправок порядка $O\left(\epsilon^{2}\right)$ в предположении, что начальные данные являются возмущениями фона (2) общего вида порядка $O(\epsilon)$ (данную постановку мы называем периодической задачей Коши для АВ), причем использовались два различных подхода: 
1) метод конечнозонного интегрирования [45]-[47], рассмотренный в статье [48] для случая одной неустойчивой моды и использованный совсем недавно для конечного числа неустойчивых мод в работе [49], который дает равномерное по пространству и времени приближение в терминах элементарных функций в главном порядке;

2) метод сращиваемых асимптотических разложений [50] для случая одной и двух неустойчивых мод, который позволяет получить удовлетворительное описание повторяемости $\mathrm{AB}$ в терминах элементарных функций.

В методе сращиваемых асимптотических разложений используется более простая математика, однако при наличии более одной неустойчивой моды он применим лишь для специальных начальных данных [49]. Отметим, что первая попытка применить конечнозонный подход для описания решений, отвечающих малым периодическим возмущениям, была предпринята в статье [51].

В простейшем случае одной неустойчивой моды $k_{1}=2 \pi / L$, где $L$ обозначает период, причем $\pi<L<2 \pi$, в работах [48], [50] было показано, что решения периодической задачи Коши для фокусирующего НУШ на интервале $[0, L]$ с начальными данными

$$
u(x, 0)=1+\epsilon(x), \quad \epsilon(x)=c_{1} e^{i k_{1} x}+c_{-1} e^{-i k_{1} x}, \quad\left|c_{1}\right|,\left|c_{-1}\right|=O(\epsilon), \quad 0<\epsilon \ll 1,
$$

можно приблизить в главном порядке следующей равномерной по пространству-времени формулой в терминах элементарных функций:

$$
\begin{gathered}
u(x, t)=\sum_{m=0}^{n} A\left(x, t ; \phi_{1}, x_{1}^{(m)}, t_{1}^{(m)}, \rho^{(m)}\right)-\frac{1-e^{4 i n \phi_{1}}}{1-e^{4 i \phi_{1}}} e^{2 i t}, \\
x \in[0, L], \quad 0 \leqslant t \leqslant t_{1}^{(n)}+O(1),
\end{gathered}
$$

где параметры $x_{1}^{(m)}, t_{1}^{(m)}, \rho^{(m)}, m \geqslant 0$, выражаются следующим образом:

$$
\begin{aligned}
& x_{1}^{(m)}=X_{1}+(m-1) \Delta X, \quad t_{1}^{(m)}=T_{1}+(m-1) \Delta T, \quad \rho^{(m)}=2 \phi_{1}+(m-1) 4 \phi_{1}, \\
& X_{1}=\frac{\arg \alpha-\phi_{1}+\pi / 2}{k_{1}}, \quad \Delta X=\frac{\arg (\alpha \beta)}{k_{1}}(\bmod L), \\
& T_{1} \equiv \frac{1}{\sigma_{1}} \ln \frac{\sigma_{1}^{2}}{2|\alpha|}, \quad \Delta T=\frac{2}{\sigma_{1}} \ln \frac{\sigma_{1}^{2}}{2 \sqrt{|\alpha \beta|}},
\end{aligned}
$$

через начальные данные

$$
\alpha=\bar{c}_{1}-e^{2 i \phi_{1}} c_{-1}, \quad \beta=\bar{c}_{-1}-e^{-2 i \phi_{1}} c_{1} .
$$

Здесь $k_{1}\left(0<k_{1}<2\right)$ и $\sigma_{1}$ обозначают волновой вектор и инкремент нарастания для неустойчивой моды соответственно:

$$
\begin{aligned}
& k_{1}=2 \cos \phi_{1} \quad \Longleftrightarrow \quad \phi_{1}=\arccos \frac{k_{1}}{2}, \\
& \sigma_{1}=\sigma\left(k_{1}\right) \equiv k_{1} \sqrt{4-k_{1}^{2}}=2 \sin \left(2 \phi_{1}\right) .
\end{aligned}
$$

Функция $A$ - это бризер Ахмедиева,

$$
\begin{gathered}
A(x, t ; \theta, X, T, \rho)=e^{2 i t+i \rho} \frac{\operatorname{ch}[\sigma(\theta)(t-T)+2 i \theta]+\sin \theta \cos [k(\theta)(x-X)]}{\operatorname{ch}[\sigma(\theta)(t-T)]-\sin \theta \cos [k(\theta)(x-X)]}, \\
k(\theta)=2 \cos \theta, \quad \sigma(\theta)=k(\theta) \sqrt{4-k^{2}(\theta)}=2 \sin (2 \theta),
\end{gathered}
$$




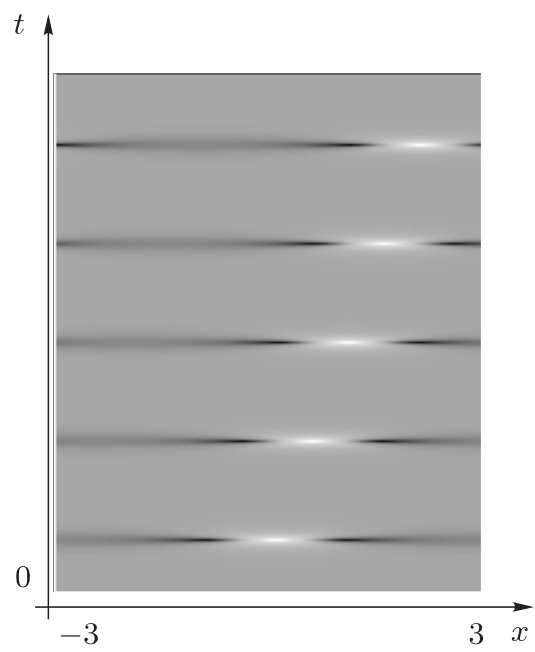

$\mathrm{a}$

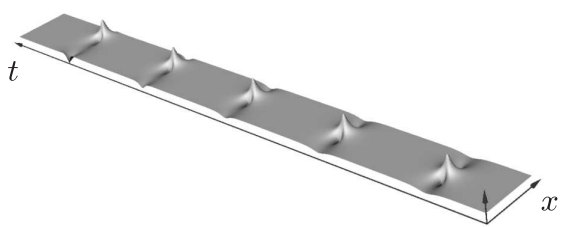

6

Рис. 1. Карта линий уровня для $|u(x, t)|$ (а) и соответствующий трехмерный график (б), показывающие чередование линейных и нелинейных стадий модуляционной неустойчивости (последовательность АВ), полученные численным интегрированием НУШ с помощью улучшенного (см. статью [52]) метода расщепления Фурье (Split Step Fourier Method) [53]-[55]. Здесь $L=6$ (присутствует одна неустойчивая мода $\left.k_{1}\right), c_{1}=\epsilon / 2, c_{-1}=\epsilon(0.3-0.4 i) / 2$, $\epsilon=10^{-4}$, короткая ось - ось $x, x \in[-L / 2, L / 2]$. Светлые области на графиках отвечают большим значениям $|u(x, t)|$. Темные области отвечают малым значениям $|u(x, t)|$. Эти графики полностью согласуются с теоретическими предсказаниями (4).

который является точным решением фокусирующего НУШ при всех вещественных значениях параметров $\theta, X, T, \rho$. Равномерное по пространству-времени представление в терминах элементарных функций имеет ошибку порядка $O\left(\epsilon^{2}\right)$ в областях, описывающих линейную стадию модуляционной неустойчивости, и ошибку порядка $O(\epsilon)$ в областях, описывающих нелинейную стадию модуляционной неустойчивости (последовательность аномальных волн), см. рис. 1.

Если вместо начального условия (3) рассмотреть периодическое возмущение общего вида

$$
\epsilon(x)=\sum_{j \geqslant 1}\left(c_{j} e^{i k_{j} x}+c_{-j} e^{-i k_{j} x}\right), \quad k_{j}=\frac{2 \pi}{L} j, \quad\left|c_{j}\right|=O(\epsilon),
$$


для случая одной неустойчивой моды $k_{1}$, последовательность АВ, описываемая формулами (4)-(9), не изменится в ведущем порядке $O(1)$, и поправка будет иметь порядок $O(\epsilon)[50]$.

Таким образом, мы имеем следующую простую картину: решение $x$-nериодической задачи Коши описывается в простейшем случае одной неустойчивой модъ точной последовательностью бризеров Ахмедиева, параметры которых, меняющиеся при каждом следующем повторении, выражаются через начальные данные в терминах элементарных функиий. Здесь $T_{1}$ - момент первого появления АВ (момент времени, в который $\mathrm{AB}$ достигает максимума абсолютного значения), $X_{1}-$ положение этого максимума, $1+2 \sin \phi_{1}$ - значение в максимуме, $\Delta T$ - время повторения (временной промежуток между двумя последовательными повторениями $\mathrm{AB}), \Delta X-x$-сдвиг максимума при повторении. Кроме этого, при каждом следующем появлении $\mathrm{AB}$ порождает изменение фона на фазовый множитель $e^{4 i \phi_{1}}$.

После того как мы показали, что общая периодическая задача Коши с одной неустойчивой модой порождает точную повторяемость АВ, описываемую солитонами Ахмедиева, возникает естественный вопрос: что произойдет, если в качестве начального условия выбрать специальное условие - солитон Ахмедиева? Поскольку гомоклинные решения неустойчивы и соответствуют в конечнозонной картине спектральным кривым, у которых все зоны закрыты, малое возмущение (возникающее, например, за счет ошибок дискретизации численной схемы для НУШ или малых поправок в силу физики процесса) приводит к открытию небольших зон, поэтому вслед за первым появлением АВ, предсказанным из начальных данных, возникает устойчивая повторяемость АВ, описываемая выражениями (4) (см. работу [56]).

Отметим также, что использование метода сращиваемых асимптотических разложений позволяет хорошо описать первое появлении АВ в другой интересной модели - РТ-симметричном НУШ (РТНУШ) [57]-[59] - с использованием семейств точных решений, недавно найденных в препринте [60], причем свободные параметры этих решений снова выражаются через начальные данные в элементарных функциях. В зависимости от начальных данных аномальные волны в РТНУШ либо регулярны, либо имеют особенность при первом появлении [61], в отличие от НУШ, АВ которого всегда регулярны.

Повторяемость АВ в периодической задаче уже изучалась в литературе (см., например, работы [62]-[67]), причем в экспериментах с волнами на воде [68], нелинейным распространением света в оптических волокнах [69] и фоторефрактивных кристаллах [70] удавалось аккуратно воспроизвести эффект повторяемости. В частности, в препринте [70] было проведено сопоставление измерений в эксперименте с вышеприведенными формулами, описывающими повторяемость в НУШ, и было получено очень хорошее качественное и количественное согласие. Мы надеемся, что хорошее согласие удастся получить и для экспериментов с оптическими волокнами, в водных бассейнах и, возможно, в бозе-конденсате с притяжением.

Общей чертой указанных экспериментов являлось использование в большинстве случаев экспериментальных установок со специальной симметрией, которая отвечает особым случаям, в которых фазовый сдвиг $\Delta X$ за одно повторение в формулах (6) равен либо нулю, либо $L / 2$, что влечет временную периодичность решений с периодом $\Delta T$ или $2 \Delta T$ соответственно, где $\Delta T$ - время повторного появления в (6). Под влиянием этих экспериментов мы посвятили оставшуюся часть работы 
исследованию АВ для начальных данных, для которых указанные симметрийные ограничения почти выполнены, и выводу полезных формул, описывающих, в частности, фазовый резонанс в повторяемости и пригодных для сопоставления как с уже доступными данными экспериментов (частично такое сопоставление было успешно проведено в препринте [70]), так и с теми, которые будут получены в ближайшем будущем.

\section{2. СИММЕТРИЧНЫЙ И КВАЗИСИММЕТРИЧНЫЙ СЛУЧАИ}

При работе в симметричном и квазисимметричном случае удобно для начала переписать формулы (4)-(7) в полярных координатах в следующем виде:

$$
\begin{gathered}
c_{1}=(|c|+\Delta) e^{i(\zeta+\delta)}, \quad c_{-1}=(|c|-\Delta) e^{i(\zeta-\delta)}, \\
\zeta=\frac{\arg c_{1}+\arg c_{-1}}{2}, \quad \delta=\frac{\arg c_{1}-\arg c_{-1}}{2}, \\
|c|=\frac{\left|c_{1}\right|+\left|c_{-1}\right|}{2}, \quad \Delta=\frac{\left|c_{1}\right|-\left|c_{-1}\right|}{2}, \quad|c|, \Delta=O(\epsilon),
\end{gathered}
$$

откуда вытекает, что

$$
\begin{gathered}
u(x, 0)=1+2\left(|c| \cos \left(k_{1} x+\delta\right)+i \Delta \sin \left(k_{1} x+\delta\right)\right) e^{i \zeta}, \\
|u(x, 0)|^{2}=1+A \cos \left(k_{1} x+\delta+B\right)+O\left(\epsilon^{2}\right), \\
A=4 \sqrt{|c|^{2} \cos ^{2} \zeta+\Delta^{2} \sin ^{2} \zeta} \quad B=\operatorname{arctg}\left(\frac{\Delta}{|c|} \operatorname{tg} \zeta\right),
\end{gathered}
$$

и

$$
\begin{aligned}
\alpha & =\left[-2 i|c| \sin \left(\phi_{1}+\zeta\right)+2 \Delta \cos \left(\phi_{1}+\zeta\right)\right] e^{i\left(\phi_{1}-\delta\right)}, \\
\beta & =\left[2 i|c| \sin \left(\phi_{1}-\zeta\right)-2 \Delta \cos \left(\phi_{1}-\zeta\right)\right] e^{-i\left(\phi_{1}-\delta\right)}, \\
\alpha \beta & =2|c|^{2}\left[\cos (2 \zeta)-\cos \left(2 \phi_{1}\right)\right]-2 \Delta^{2}\left[\cos (2 \zeta)+\cos \left(2 \phi_{1}\right)\right]+2 i \Delta|c| \sigma_{1} .
\end{aligned}
$$

Отметим, что в случае, когда мы возбуждаем только единственную неустойчивую моду, фазовый сдвиг $\delta$ отвечает тривиальному сдвигу по $x$ начальных условий, поэтому, используя трансляционную симметрию НУШ, можно без потери общности положить $\delta=0$, что мы и делаем ниже.

2.1. Симметричный случай $\left|c_{1}\right|=\left|c_{-1}\right| \Longleftrightarrow \Delta=0$. Для начала отметим, что из формул (6) и (13) видно, что эксперименты [68]-[70] по повторяемости АВ, в которых сдвиг по $x$ при повторении оказывается чаще всего равным $\Delta X=0$ или $\Delta X=L / 2$, отвечают специальному выбору параметров, для которого $\alpha \beta \in \mathbb{R}$ или, что эквивалентно, $\Delta=0$ :

$$
\alpha \beta \in \mathbb{R} \Longleftrightarrow \Delta=0 \Longleftrightarrow\left|c_{1}\right|=\left|c_{-1}\right| .
$$

В этих двух важных случаях (см. рис. 2)

$$
\begin{gathered}
\Delta X=0 \Longleftrightarrow \alpha \beta>0 \Longleftrightarrow \cos (2 \zeta)>\cos \left(2 \phi_{1}\right) \Longleftrightarrow|\zeta|<\phi_{1}, \\
\pi-\phi_{1}<|\zeta| \leqslant \pi, \\
\Delta X=\frac{L}{2} \Longleftrightarrow \alpha \beta<0 \Longleftrightarrow \cos (2 \zeta)<\cos \left(2 \phi_{1}\right) \Longleftrightarrow \phi_{1}<|\zeta|<\pi-\phi_{1},
\end{gathered}
$$




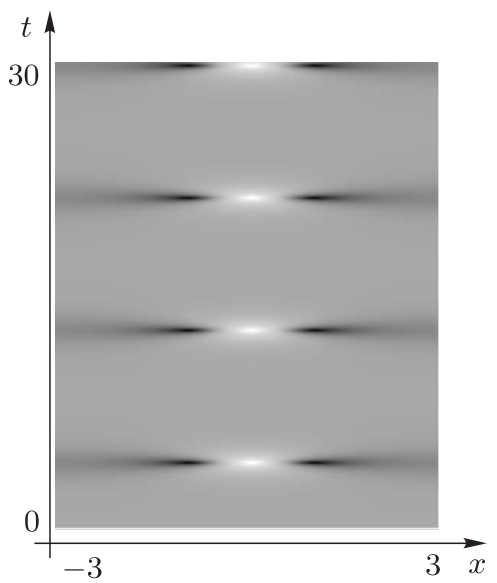

a

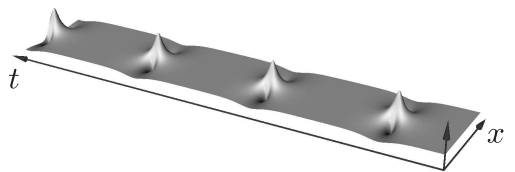

B

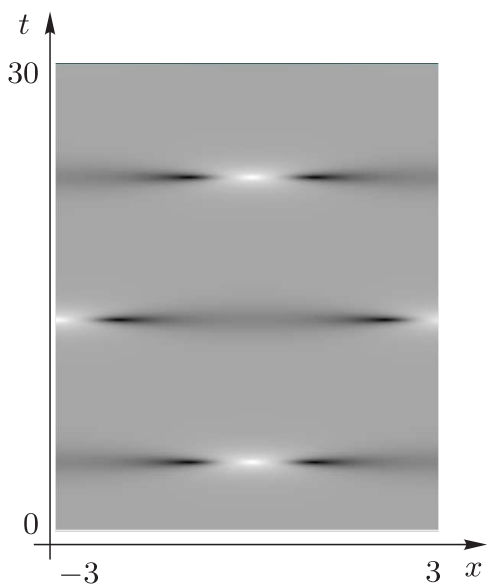

б

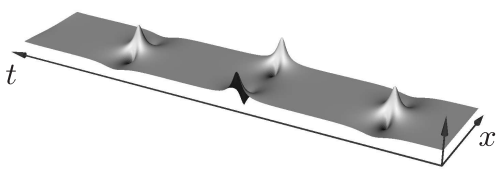

$\Gamma$

Рис. 2. Две карты линий уровня для $|u(x, t)|(\mathrm{a}, б)$ и соответствующие трехмерные графики (в, г), полученные численным интегрированием НУШ, описывающие повторяемость АВ в симметричном случае $\Delta=0$ с $L=6$ $\left(k_{1} \sim 1.047\right.$ и $\left.\phi_{1} \sim 1.020\right),\left|c_{1}\right|=\left|c_{-1}\right|=0.5 \epsilon, \epsilon=10^{-3}$. На рис. а, в $\zeta=0.3<\phi_{1}$, соответственно $\Delta X=0$. На рис. б, г $\phi_{1}<\zeta=1.3<\pi-\phi_{1}$, соответственно $\Delta X=L / 2$. Светлым на графиках показаны большие значения $|u(x, t)|$, темным - малые значения $|u(x, t)|$. Мы имеем полное согласие с теорией (4).

причем

$$
\begin{gathered}
\alpha=-2 i|c| \sin \left(\phi_{1}+\zeta\right) e^{i \phi_{1}}, \quad \beta=2 i|c| \sin \left(\phi_{1}-\zeta\right) e^{-i \phi_{1}}, \\
\alpha \beta=2|c|^{2}\left(\cos (2 \zeta)-\cos \left(2 \phi_{1}\right)\right)
\end{gathered}
$$

(здесь мы воспользовались элементарным соотношением

$$
\left.2 \sin \left(\phi_{1}+\zeta\right) \sin \left(\phi_{1}-\zeta\right)=\cos (2 \zeta)-\cos \left(2 \phi_{1}\right)\right)
$$

В резонансных фазах $\zeta=-\phi_{1}, \pi-\phi_{1}, \alpha=0$, поэтому величина $T_{1}$ обращается в бесконечность, а $X_{1}$ имеет разрыв. Аналогично, в резонансных фазах $\zeta=$ $\pm \phi_{1}, \pm\left(\pi-\phi_{1}\right), \alpha \beta=0$, величина $\Delta T$ обращается в бесконечность, а $\Delta X$ имеет разрыв. Особое поведение связано с тем, что формулы (6) представляют собой лишь первый член соответствующего асимптотического ряда [48], и вблизи указанных точек нужно учитывать следующие поправки. Вместо этого ниже мы исследуем регуляризацию, порождаемую тем обстоятельством, что соотношение симметрии начальных данных $\Delta=0 \Longleftrightarrow\left|c_{1}\right|=\left|c_{-1}\right|$ (3) в реальных экспериментах выполнено лишь приблизительно. 
2.2. Квазисимметричный случай $\left|c_{1}\right| \sim\left|c_{-1}\right|$. Начиная с данного момента мы работаем с квазисимметричной конфигурацией

$$
\left|c_{1}\right| \sim\left|c_{-1}\right|=O(\epsilon), \quad O\left(\epsilon^{2}\right) \ll|\Delta| \ll O(\epsilon) .
$$

Неравенства в формуле (17) вытекают из того, что величина $|\Delta|$ должна быть существенно меньше, чем $\left|c_{1}\right|,\left|c_{-1}\right|=O(\epsilon)$, и больше, чем поправка $O\left(\epsilon^{2}\right)$, возникающая в разложении по малому возмущению, порождающему (6).

При указанных предположениях формулы (12) и (13) существенно упрощаются:

$$
\begin{aligned}
& u(x, 0)=1+2|c| \cos \left(k_{1} x\right) e^{i \zeta}+O(\Delta), \quad|u(x, 0)|^{2}=1+4|c||\cos \zeta| \cos \left(k_{1} x\right)+O(\Delta), \\
& \alpha=\left(-2 i|c| \sin \left(\phi_{1}+\zeta\right)+2 \Delta \cos \left(\phi_{1}+\zeta\right)\right) e^{i \phi_{1}} \sim \\
& \sim \begin{cases}-2 i|c| \sin \left(\phi_{1}+\zeta\right) e^{i\left(\phi_{1}-\delta\right)}, & \left|\zeta+\phi_{1}\right|,\left|\zeta-\pi+\phi_{1}\right|=O(1), \\
2 \Delta e^{i \phi_{1}}, & \zeta=-\phi_{1}, \\
-2 \Delta e^{i \phi_{1}}, & \zeta=\pi-\phi_{1},\end{cases} \\
& \beta=\left(2 i|c| \sin \left(\phi_{1}-\zeta\right)-2 \Delta \cos \left(\phi_{1}-\zeta\right)\right) e^{-i \phi_{1}} \sim \\
& \sim\left\{\begin{array}{cl}
2 i|c| \sin \left(\phi_{1}-\zeta\right) e^{-i \phi_{1}}, & \left|\zeta-\phi_{1}\right|,\left|\zeta+\pi-\phi_{1}\right|=O(1), \\
-2 \Delta e^{-i \phi_{1}}, & \zeta=\phi_{1}, \\
2 \Delta e^{-i \phi_{1}}, & \zeta=-\pi+\phi_{1},
\end{array}\right. \\
& \alpha \beta=2|c|^{2}\left(\cos (2 \zeta)-\cos \left(2 \phi_{1}\right)\right)+2 i \sigma_{1}|c| \Delta+O\left(\Delta^{2}\right) \sim \\
& \sim \begin{cases}2|c|^{2}\left(\cos (2 \zeta)-\cos \left(2 \phi_{1}\right)\right), & \left|\zeta \mp \phi_{1}\right|,\left|\zeta \mp\left(\pi-\phi_{1}\right)\right|=O(1), \\
2 i \sigma_{1}|c| \Delta, & \zeta= \pm \phi_{1}, \pm\left(\pi-\phi_{1}\right) .\end{cases}
\end{aligned}
$$

Отсюда следует, что для момента появления первой АВ мы имеем соотношение

$$
\begin{aligned}
T_{1} & \sim \frac{1}{\sigma_{1}} \ln \left(\frac{\sigma_{1}^{2}}{4 \sqrt{|c|^{2} \sin ^{2}\left(\phi_{1}+\zeta\right)+\Delta^{2} \cos ^{2}\left(\phi_{1}+\zeta\right)}}\right) \sim \\
& \sim \begin{cases}\frac{1}{\sigma_{1}} \ln \left(\frac{\sigma_{1}^{2}}{4|c|\left|\sin \left(\phi_{1}+\zeta\right)\right|}\right), & \left|\zeta+\phi_{1}\right|,\left|\zeta-\pi+\phi_{1}\right|=O(1), \\
\frac{1}{\sigma_{1}} \ln \left(\frac{\sigma_{1}^{2}}{4|\Delta|}\right), & \zeta=-\phi_{1}, \pi-\phi_{1} .\end{cases}
\end{aligned}
$$

Если зафиксировать $k_{1}\left(\phi_{1}\right)$ и $|c|$, величина $T_{1}$ становится функцией единственной переменной $\zeta$, и эта функция имеет максимумы в резонансных фазах $\zeta=-\phi_{1}, \pi-\phi_{1}$ со значением

$$
T_{1}\left(-\phi_{1}\right)=\frac{1}{\sigma_{1}} \ln \left(\frac{\sigma_{1}^{2}}{4|\Delta|}\right)
$$

и минимумы при $\zeta=\pi / 2-\phi_{1}$ и $\zeta=-\pi / 2-\phi_{1}$ со значением

$$
T_{1}\left(\frac{\pi}{2}-\phi_{1}\right)=\frac{1}{\sigma_{1}} \ln \left(\frac{\sigma_{1}^{2}}{4|c|}\right)
$$

(см. график с двумя пиками на рис. 3). Соответственно $X_{1}$ приблизительно равно $\operatorname{sgn} \Delta L / 2$ на интервале $-\pi \leqslant \zeta<-\phi_{1}$ с резким переходом от $\operatorname{sgn} \Delta L / 2$ к нулю на 


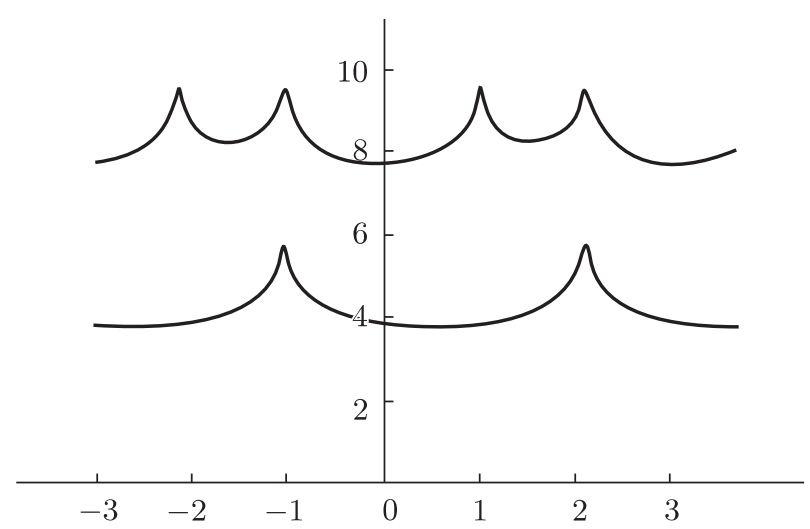

Рис. 3. Графики момента первого появления $T_{1}$ (кривая с двумя максимумами) и интервала между последующими появлениями $\Delta T$ (кривая с четырьмя максимумами) как функций от переменной $\zeta$ для $|c|=10^{-3}, \Delta=|c|^{3 / 2}$, $k_{1}=2 \pi / L=1.0472$ (и $\left.\phi_{1}=1.0197\right)$. График $T_{1}$ имеет два максимума в резонансах $\zeta=-\phi_{1}$ и $\zeta=\pi-\phi_{1} \sim 2.12$ и два минимума при $\zeta=\pi / 2-\phi_{1} \sim 0.55$ и $\zeta=-\pi / 2-\phi_{1} \sim-2.59$. График $\Delta T$ имеет четыре максимума в резонансах $\zeta= \pm \phi_{1}$ и $\zeta= \pm\left(\pi-\phi_{1}\right) \sim \pm 2.12$ и глобальный минимум при $\zeta=0$.

интервале порядка $O(|\Delta| /|c|)$, когда $\zeta$ проходит в положительном направлении резонансную точку $-\phi_{1}$. Далее оно почти постоянно на интервале $-\phi_{1}<\zeta<\pi-\phi_{1}$ с резким переходом того же типа от нуля к $-\operatorname{sgn} \Delta L / 2$, когда $\zeta$ проходит в положительном направлении резонансную точку $\pi-\phi_{1}$. Наконец, оно почти постоянно на интервале $\pi-\phi_{1}<\zeta \leqslant \pi$. Отметим, что $X_{1}=\operatorname{sgn} \Delta L / 4$ при $\zeta=-\phi_{1}$ и $X_{1}=-\operatorname{sgn} \Delta L / 4$ при $\zeta=\pi-\phi_{1}$. Заметим также, что поскольку $X_{1}$ определено по $\bmod L$, то $X_{1}= \pm L / 2$ отвечает одной и той же конфигурации.

Можно выписать аналогичные формулы для $\Delta T$ и $\Delta X$ :

$$
\begin{aligned}
\Delta T & \sim \frac{2}{\sigma_{1}} \ln \left(\frac{\sigma_{1}^{2}}{2 \sqrt{2|c|} \sqrt[4]{|c|^{2}\left(\cos (2 \zeta)-\cos \left(2 \phi_{1}\right)\right)^{2}+\Delta^{2} \sigma_{1}^{2}}}\right) \sim \\
& \sim \begin{cases}\frac{2}{\sigma_{1}} \ln \left(\frac{\sigma_{1}^{2}}{2 \sqrt{2}|c| \sqrt{\left|\cos (2 \zeta)-\cos \left(2 \phi_{1}\right)\right|}}\right), & \left|\zeta \mp \phi_{1}\right|,\left|\zeta \mp\left(\pi-\phi_{1}\right)\right|=O(1) \\
\frac{2}{\sigma_{1}} \ln \left(\frac{\sigma_{1}^{2}}{2 \sqrt{2 \sigma_{1}|c||\Delta|}}\right), & \zeta= \pm \phi_{1}, \pm\left(\pi-\phi_{1}\right)\end{cases} \\
\Delta X & \sim \frac{1}{k_{1} \arg \left(\left(\cos (2 \zeta)-\cos \left(2 \phi_{1}\right)\right)+i \frac{\Delta}{|c|} \sigma_{1}\right) \sim}, \quad \cos (2 \zeta)>\cos \left(2 \phi_{1}\right), \\
& \sim \begin{cases}\frac{\sigma_{1} \Delta}{k_{1}|c|\left(\cos (2 \zeta)-\cos \left(2 \phi_{1}\right)\right)} \sim 0, & \cos (2 \zeta)<\cos \left(2 \phi_{1}\right), \\
\operatorname{sgn} \Delta \frac{L}{2}-\frac{\sigma_{1} \Delta}{k_{1}|c|\left(\cos (2 \zeta)-\cos \left(2 \phi_{1}\right)\right)} \sim \operatorname{sgn} \Delta \frac{L}{2}, & \zeta= \pm \phi_{1}, \pm\left(\pi-\phi_{1}\right) . \\
\operatorname{sgn} \Delta \frac{L}{4}, & \end{cases}
\end{aligned}
$$



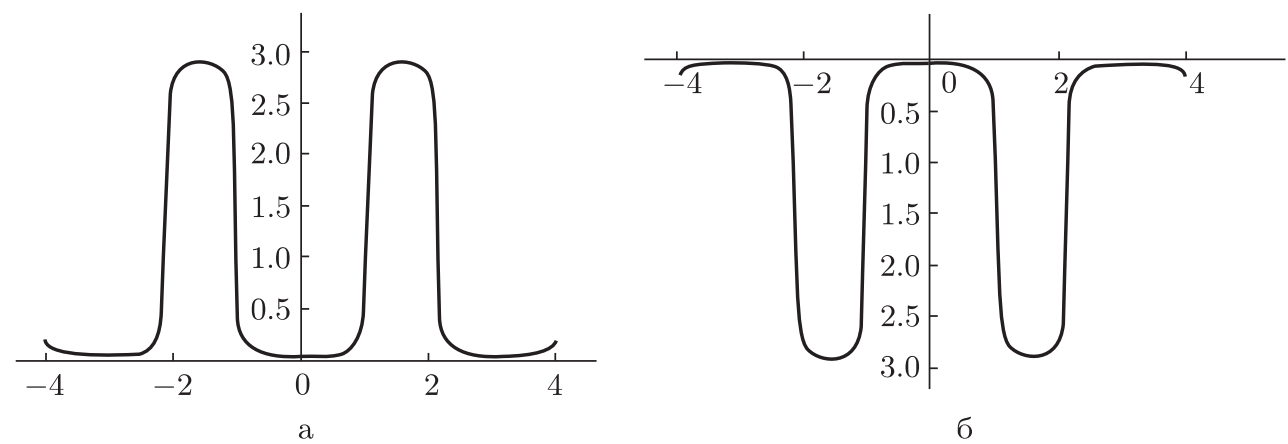

Рис. 4. Графики $x$-сдвигов $\Delta X$ при повторных появлениях в зависимости от переменной $\zeta$ для $k_{1}=2 \pi / L=1.0472\left(\phi_{1}=1.0197\right)$ и $|c|=10^{-3}$ при $\Delta=|c|^{3 / 2} \sim 3.16 \cdot 10^{-5}$ (а) и $\Delta=-|c|^{3 / 2}$ (б).
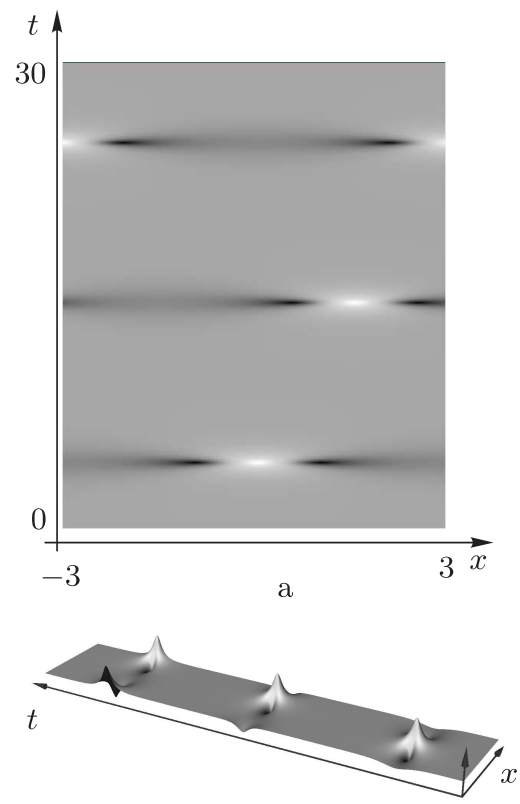

B
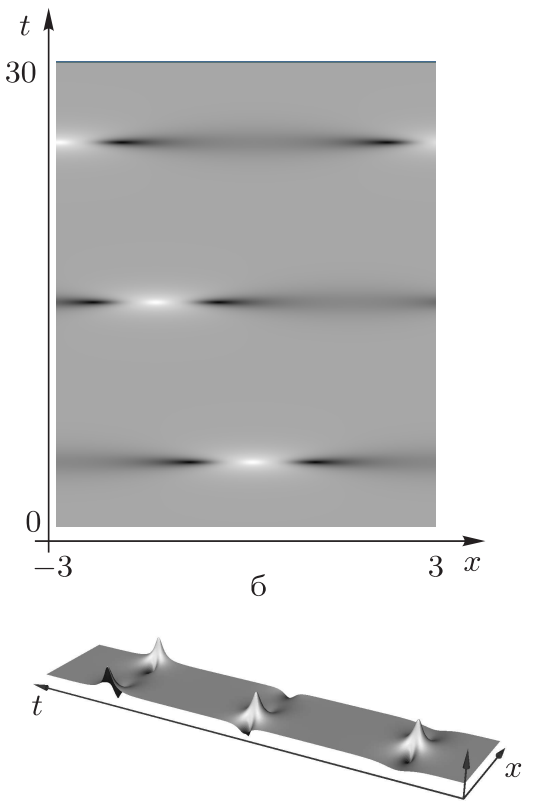

$\Gamma$

Рис. 5. Две карты линий уровня для $|u(x, t)|(\mathrm{a}, \sigma)$ и соответствующие трехмерные графики (в,г), описывающие повторяемость АВ в квазисимметричном случае для резонанса $\arg c_{1}=\arg c_{-1}=\phi_{1}$, полученные численным интегрированием НУШ для $L=6\left(k_{1}=1.0472\right.$ и $\left.\phi_{1}=1.0197\right)$. На рис. а, в $\left|c_{1}\right|=0.515 \epsilon,\left|c_{-1}\right|=0.485 \epsilon, \epsilon=10^{-3}$. Поскольку $\Delta=0.015 \epsilon>0$, то $\Delta X=L / 4$. На рис. б, г $\left|c_{1}\right|=0.485 \epsilon,\left|c_{-1}\right|=0.515 \epsilon, \epsilon=10^{-3}$. Светлым на графиках показаны большие значения $|u(x, t)|$, темным - малые значения $|u(x, t)|$. Эти графики полностью согласуются с изложенной выше теорией. 
Отсюда следует, что величина $\Delta T$, рассматриваемая как функция фазы $\zeta$, имеет максимумы в резонансных фазах $\zeta= \pm \phi_{1}, \pm\left(\pi-\phi_{1}\right)$ со значением

$$
\Delta T\left( \pm \phi_{1}\right) \sim \frac{2}{\sigma_{1}} \ln \left(\frac{\sigma_{1}^{2}}{2 \sqrt{2 \sigma_{1}|c||\Delta|}}\right)
$$

и глобальный минимум при $\zeta=0$ со значением

$$
\Delta T(0) \sim \frac{2}{\sigma_{1}} \ln \left(\frac{\sigma_{1}^{2}}{2 \sqrt{2}|c| \sqrt{1-\cos \left(2 \phi_{1}\right)}}\right)
$$

(см. график с четырьмя пиками на рис. 3).

Соответственно $\Delta X$ приблизительно равно нулю при $0 \leqslant \zeta<\phi_{1}$, резко изменяясь от нуля до $\operatorname{sgn} \Delta L / 2$ на малом интервале порядка $O(2|\Delta| /|c|)$, когда $\zeta$ проходит в положительном направлении через резонанс $\phi_{1}$, далее оно почти постоянно на интервале $\phi_{1}<\zeta<\pi-\phi_{1}$ и снова резко изменяется от $\operatorname{sgn} \Delta L / 2$ до нуля, когда $\zeta$ проходит в положительном направлении через резонанс $\pi-\phi_{1}$. Наконец, оно остается почти постоянным на интервале $\pi-\phi_{1}<\zeta \leqslant \pi$. Кроме того, в резонансных фазах $\phi_{1}$ и $\pi-\phi_{1}$ имеет место равенство $\Delta X=\operatorname{sgn} \Delta L / 4$. Поведение при отрицательных $\zeta$ следует из симметрии $\Delta X$ по отношению к $\zeta$ (см. графики $\Delta X(\zeta)$ для положительных и отрицательных значениях $\Delta$ на рис. 4 , а также результаты численного интегрирования НУШ в резонансной точке $\zeta=\phi_{1}$ на рис. 5).

\section{3. ЗАКЛЮЧИТЕЛЬНЫЕ ЗАМЕЧАНИЯ}

В настоящей работе мы детально изучили фазовые резонансы для повторяемости АВ в модели НУШ в квазисимметричном случае, который имеет приложения к ряду практических задач, включая волны на воде [68], в волоконной оптике [69] и фоторефрактивных кристаллах [70]. В частности, в препринте [70] был использован симметричный трехволновой интерферометр для генерации фоновой волны и одномодового возмущения вида (18), распространяющихся в фоторефрактивных кристаллах (переменная $t$ в уравнении НУШ в данном случае соответствует расстоянию, пройденному волной в кристалле), а условие симметрии (17) отражало тот факт, что боковые пучки имеют почти равную интенсивность. Резонанс появляется, когда средняя величина $\zeta$ для фаз двух боковых пучков (измеренная относительно центрального пучка) равна $\pm \phi_{1}, \pm\left(\pi-\phi_{1}\right)$, где $\phi_{1}=\arccos \left(k_{1} / 2\right)$ и $k_{1}$ - мода, возбуждаемая начальным возмущением.

Формулы, выведенные в данной работе, во-первых, можно сопоставить с экспериментальными данными, доступными в настоящее время (частично такое сравнение было проведено в работе [70]), или теми, которые будут получены в ближайщем будущем, что позволит, в частности, проверить, насколько точно интегрируемая модель НУШ описывает физику изучаемых процессов; во-вторых, их можно использовать, в частности, для восстановления точных начальных данных по экспериментальным измерениям параметров повторяемости (то, что мы называем обратной задачей в статье [50], см. также препринт [70]).

Благодарности. Авторы благодарны Е. Дель Ре, Д. Пьеранжели и С. Трилло за полезные обсуждения. 


\section{Список литературы}

[1] В. Е. Захаров, "Устойчивость периодических волн конечной амплитуды на поверхности глубокой жидкости", ПМТФ, 9:2 (1968), 86-94.

[2] D. R. Solli, C. Ropers, P. Koonath, B. Jalali, "Optical rogue waves", Nature, 450:7172 (2007), 1054.

[3] U. Bortolozzo, A. Montina, F. T. Arecchi, J. P. Huignard, S. Residori, "Spatiotemporal pulses in a liquid crystal optical oscillator", Phys. Rev. Lett., 99:2 (2007), 023901, 4 pp.

[4] D. Pierangeli, F. Di Mei, C. Conti, A. J. Agranat, E. DelRe, "Spatial rogue waves in photorefractive ferroelectrics", Phys. Rev. Lett., 115:9 (2015), 093901, 6 pp.

[5] C. Sulem, P-L. Sulem, The Nonlinear Schrödinger Equation. Self Focusing and Wave Collapse, Applied Mathematical Sciences, 139, Springer, New York, 1999.

[6] Yu. V. Bludov, V. V. Konotop, N. Akhmediev, "Matter rogue waves", Phys. Rev. A, 80:3 (2009), 033610, 5 pp.

[7] G. G. Stokes, "On the theory of oscillatory waves", Mathematical and Physical Papers, Transactions of the Cambridge Philosophical Society, VIII, Cambridge Univ. Press, Cambridge, 2009, 441-455; "Supplement to a paper on the theory of oscillatory waves", Mathematical and Physical Papers, v. 1, Cambridge Univ. Press, Cambridge, 1880, 314-326.

[8] В. И. Беспалов, В.И. Таланов, "О нитевидной структуре пучков света в нелинейных жидкостях", Писъма в ЖЖЭТФ, 3:12 (1966), 471-476.

[9] T. B. Benjamin, J.E. Feir, "The disintegration of wave trains on deep water. Part I. Theory", J. Fluid Mech., 27 (1967), 417-430.

[10] V. Zakharov, L. Ostrovsky, "Modulation instability: the beginning", Phys. D, 238:5 (2009), $540-548$.

[11] T. Taniuti, H. Washimi, "Self-trapping and instability of hydromagnetic waves along the magnetic field in a cold plasma", Phys. Rev. Lett., 21:4 (1968), 209-212.

[12] L. Salasnich, A. Parola, L. Reatto, "Modulational instability and complex dynamics of confined matter-wave solitons", Phys. Rev. Lett., 91:8 (2003), 080405, 4 pp., arXiv: cond-mat/0307206.

[13] K. L. Henderson, D. H. Peregrine, J. W. Dold, "Unsteady water wave modulations: fully nonlinear solutions and comparison with the nonlinear Schrödinger equation", Wave Motion, 29:4 (1999), 341-361.

[14] K. B. Dysthe, K. Trulsen, "Note on breather type solutions of the NLS as models for freak-waves", Phys. Scr., T82:1 (1999), 48-52.

[15] A. Osborne, M. Onorato, M. Serio, "The nonlinear dynamics of rogue waves and holes in deep-water gravity wave trains", Phys. Lett. A, 275:5-6 (2000), 386-393.

[16] C. Kharif, E. Pelinovsky, "Physical mechanisms of the rogue wave phenomenon", Eur. J. Mech. B Fluids, 22:6 (2004), 603-634.

[17] C. Kharif, E. Pelinovsky, T. Talipova, A. Slunyaev, "Focusing of nonlinear wave groups in deep water", Писъма в ЖЭЭТФ, 73:4 (2001), 190-195.

[18] M. Onorato, S. Residori, U. Bortolozzo, A. Montina, F. T. Arecchi, "Rogue waves and their generating mechanisms in different physical contexts", Phys. Rep., 528:2 (2013), 47-89.

[19] В. Е. Захаров, А. Б. Шабат, “Точная теория двумерной самофокусировки и одномерной автомодуляции волн в нелинейных средах", ЖЭТФ, 61:1 (1971), 118-134.

[20] А. Р. Итс, А. В. Рыбин, М. А. Салль, "К вопросу о точном интегрировании нелинейного уравнения Шредингера", ТМФ, 74:1 (1988), 29-45.

[21] E. D. Belokolos, A. I. Bobenko, V.Z. Enol'ski, A. R. Its, V. B. Matveev, Algebro-Geometric Approach in the Theory of Integrable Equations, Springer Series in Nonlinear Dynamics, Springer, Berlin, 1994.

[22] И. М. Кричевер, "Спектральная теория двумерных периодических операторов и ее приложения", УМН, 44:2(266) (1989), 121-184. 
[23] I. M. Krichever, "Perturbation theory in periodic problems for two-dimensional integrable systems", Sov. Sci. Rev., Sect. C, Math. Phys. Rev., 9:2 (1992), 1-103.

[24] V.B. Matveev, M. A. Salle, Darboux Transformations and Solitons, Springer, Berlin, 1991.

[25] N. Ercolani, M. G. Forest, D. W. McLaughlin, "Geometry of the modulation instability Part III: homoclinic orbits for the periodic sine-Gordon equation", Phys. D, 43:2-3 (1990), 349-384.

[26] В. Е. Захаров, А. Б. Шабат, "Схема интегрирования нелинейных уравнений математической физики методом обратной задачи рассеяния. I", Функи. анализ и его прил., 8:3 (1974), 43-53.

[27] В. Е. Захаров, А. В. Михайлов, "Релятивистски-инвариантные двумерные модели теории поля, интегрируемые методом обратной задачи", ЖЭТФ, 74:6 (1978), 1953-1973.

[28] D.H. Peregrine, "Water waves, nonlinear Schrödinger equations and their solutions", J. Austral. Math. Soc. Ser. B, 25:1 (1983), 16-43.

[29] Е. А. Кузнецов, "Солитоны в параметрически неустойчивой плазме”, Докл. АН СССР, 236:1-3 (1977), 575-577.

[30] T. Kawata, H. Inoue, "Inverse scattering method for the nonlinear evolution equations under nonvanishing conditions", J. Phys. Soc. Japan, 44:5 (1978), 1722-1729.

[31] Y.-C. Ma, "The perturbed plane wave solutions of the cubic Schrödinger equation", Stud. Appl. Math., 60:1 (1979), 43-58.

[32] Н. Н. Ахмедиев, В. М. Елеонский, Н.Е. Кулагин, "Генерация периодических пакетов пикосекундных импульсов в оптическом фибере: точные решения", ЖЭТФ, 89 (1985), $1542-1551$.

[33] Н. Н. Ахмедиев, В. И. Корнеев, "Модуляционная неустойчивость и периодические решения нелинейного уравнения Шредингера", ТМФ, 69:2 (1986), 189-194.

[34] Н. Н. Ахмедиев, В. М. Елеонский, Н. Е. Кулагин, “Точные решения первого порядка нелинейного уравнения Шредингера", ТМФ, 72:2 (1987), 183-196.

[35] V.E. Zakharov, A. A. Gelash, Soliton on unstable condensate, arXiv: 1109.0620.

[36] P. Dubard, P. Gaillard, C. Klein, V. B. Matveev, Eur. Phys. J. Special Topics, 185:1 (2010), $247-258$.

[37] R. Hirota, Direct Methods for Finding Exact Solutions of Nonlinear Evolution Equations, Lecture Notes in Mathematics, 515, Springer, New York, 1976.

[38] D. J. Kedziora, A. Ankiewicz, N. Akhmediev, "Second-order nonlinear Schrödinger equation breather solutions in the degenerate and rogue wave limits", Phys. Rev. E, 85:6 (2012), 066601, 9 pp.

[39] V.E. Zakharov, A. A. Gelash, "On the nonlinear stage of modulation instability", Phys. Rev. Lett., 111:5 (2013), 054101, 5 pp.

[40] F. Baronio, A. Degasperis, M. Conforti, S. Wabnitz, "Solutions of the vector nonlinear Schrödinger equations: evidence for deterministic rogue waves", Phys. Rev. Lett., 109:4 (2012), 044102, 4 pp.

[41] A. Degasperis, S. Lombardo, "Integrability in action: solitons, instability and rogue waves", Rogue and Shock Waves in Nonlinear Dispersive Media, Lecture Notes in Physics, 926, eds. M. Onorato, S. Resitori, F. Baronio, Springer, Cham, 2016.

[42] A. Degasperis, S. Lombardo, M. Sommacal, "Integrability and linear stability of nonlinear waves", J. Nonlinear Sci., 2018, 1-41, arXiv: 1707.09536.

[43] G. Biondini, G. Kovacic, "Inverse scattering transform for the focusing nonlinear Schrödinger equation with nonzero boundary conditions", J. Math. Phys., 55:3 (2014), 031506, 22 pp.

[44] G. Biondini, S. Li, D. Mantzavinos, "Oscillation structure of localized perturbations in modulationally unstable media", Phys. Rev. E, 94:6 (2016), 060201, 6 pp.

[45] С. П. Новиков, "Периодическая задача для уравнения Кортевега-де Фриза. I", Функи. анализ и его прил., 8:3 (1974), 54-66. 
[46] А. Р. Итс, В. П. Котляров, “Явные формулы для решения нелинейного уравнения Шредингера", Докл. АН УССР. Сер. А, 11 (1976), 965-968, 1051.

[47] И. М. Кричевер, “Методы алгебраической геометрии в теории нелинейных уравнений”, УМH, 32:6(198) (1977), 183-208.

[48] P. G. Grinevich, P. M. Santini, The finite gap method and the analytic description of the exact rogue wave recurrence in the periodic NLS Cauchy problem. 1, arXiv: 1707.05659.

[49] P. G. Grinevich, P. M. Santini, The finite gap method and the solution of the rogue wave periodic Cauchy problem in the case of a finite number of unstable modes, Preprint, 2018, in preparation.

[50] P. G. Grinevich, P. M. Santini, "The exact rogue wave recurrence in the NLS periodic setting via matched asymptotic expansions, for 1 and 2 unstable modes", Phys. Lett. A, 382:14 (2018), 973-979, arXiv: 1708.04535.

[51] E. R. Tracy, H.H. Chen, "Nonlinear self-modulation: an exactly solvable model", Phys. Rev. A, 37:3 (1988), 815-839.

[52] J. Javanainen, J. Ruostekoski, "Symbolic calculation in development of algorithms: split-step methods for the Gross-Pitaevskii equation", J. Phys. A: Math. Gen., 39:12 (2006), L179-L184, arXiv: cond-math/0411154.

[53] G. P. Agrawal, Nonlinear Fiber Optics, Academic Press, New York, 2001.

[54] J.A. C. Weideman, B. M. Herbst, "Split-step methods for the solution of the nonlinear Schrödinger equation", SIAM J. Numer. Anal., 23:3 (1986), 485-507.

[55] T. R. Taha, X. Xu, "Parallel split-step fourier methods for the coupled nonlinear Schrödinger type equations", J. Supercomp., 32:1 (2005), 5-23.

[56] P. G. Grinevich, P. M. Santini, Numerical instability of the Akhmediev breather and a finite-gap model of it, arXiv: 1708.00762; Recent Developments in Integrable Systems and Related Topics of Mathematical Physics, eds. V. M. Buchstaber, S. Konstantinou-Rizo, A. V. Mikhailov, Springer, New York, 2018 (to appear).

[57] M. J. Ablowitz, Z.H. Musslimani, "Integrable nonlocal nonlinear Schrödinger equation", Phys. Rev. Lett., 110:8 (2013), 064105, 5 pp.

[58] M. J. Ablowitz, Z. H. Musslimani, "Integrable discrete PT symmetric model", Phys. Rev. Lett. E, 90:3 (2014), 032912, 5 pp.

[59] M. J. Ablowitz, Z.H. Musslimani, "Integrable nonlocal nonlinear equations", Stud. Appl. Math., 139:1 (2017), 7-59.

[60] P. M. Santini, Darboux-Dressing and symmetry construction of classes of regular and singular solutions of the NLS and the PT-symmetric NLS equations over the constant background, Preprint, 2018.

[61] P. M. Santini, The first rogue wave appearance in the rogue wave periodic Cauchy problem for the PT-symmetric NLS: regular dynamics or blow up at finite time, Preprint, 2018.

[62] H. C. Yuen, W. E. Ferguson, "Relationship between Benjamin-Feir instability and recurrence in the nonlinear Schrödinger equation", Phys. Fluids, 21:8 (1978), 1275-1278.

[63] B. M. Lake, H. C. Yuen, H. Rungaldier, W. E. Ferguson, "Nonlinear deep-water waves: Theory and experiment. Part 2. Evolution of a continuous wave train", J. Fluid Mech., 83:1 (1977), 49-74.

[64] H. Yuen, B. Lake, "Nonlinear dynamics of deep-water gravity waves", Adv. Appl. Mech., 22 (1982), 67-229.

[65] N. N. Akhmediev, "Nonlinear physics: Déjà vu in optics", Nature, 413 (2001), 267-268.

[66] G. Van Simaeys, P. Emplit, M. Haelterman, "Experimental demonstration of the Fermi-Pasta-Ulam recurrence in a modulationally unstable optical wave", Phys. Rev. Lett., 87:3 (2001), 033902, 4 pp.

[67] E. A. Kuznetsov, "Fermi-Pasta-Ulam recurrence and modulation instability", Писъма в ЖЭТФ, 105:2 (2017), 108-109. 
[68] O. Kimmoun, H. C. Hsu, H. Branger, M.S. Li, Y.Y. Chen, C. Kharif, M. Onorato, E. J. R. Kelleher, B. Kibler, N. Akhmediev, A. Chabchoub, "Modulation instability and phase-shifted Fermi-Pasta-Ulam recurrence", Sci. Rep., 6 (2016), 28516, 9 pp.

[69] A. Mussot, C. Naveau, M. Conforti, A. Kudlinski, P. Szriftgiser, F. Copie, S. Trillo, "Fibre multiwave-mixing combs reveal the broken symmetry of Fermi-Pasta-Ulam recurrence", Nature Photonics, 12 (2018), 303-308.

[70] D. Pierangeli, M. Flammini, L. Zhang, G. Marcucci, A. J. Agranat, P. G. Grinevich, P. M. Santini, C. Conti, E. DelRe, Observation of exact Fermi-Pasta-Ulam recurrence, Preprint, 2018. 